\title{
Cultura y praxis comunicacional Pertinencia de lo cultural en el diseño y aplicación de diagnósticos de comunicación
}

\begin{abstract}
Resumen
Estudia la relación entre la cultura y la comunicación en el análisis, diseño e implementación de los diagnósticos de la comunicación. Enfatiza en el propósito del diagnóstico en comunicación, que es conocer la realidad para superar la situación de una institución o grupo social, además, señala las diferentes perspectivas y sus consecuencias cuando no se elabora un diagnóstico apropiado. Así, informa sobre la existencia de varias teorías para la aplicación social que requieren que el diagnóstico en comunicación les permita identificar desde los actores y sus percepciones, los conocimientos, el nivel de información, las actitudes, las prácticas y los componentes; también, se refiere a la flexibilidad como característica importante durante la planificación del diagnóstico, de manera que pueda ajustarse a la realidad.
\end{abstract}

Palabras clave: Comunicación social, diagnóstico, cultura, planificación, aplicación.

\author{
CULTURE AND COMMUNICATIVE PRAXIS \\ RELEVANCE OF CULTURE IN THE DESIGN AND \\ IMPLEMENTATION OF COMMUNICATION DIAGNOSTICS
}

\begin{abstract}
Study the relationship between culture and communication in the analysis, desing and implementation of diagnostic communication. Emphasizes the purpose of diagnosis in communication, it is to know reality to overcome the situation of an institution or social group. Also, points out the different perspectives and their consequences when not made a proper diagnosis. So, it reports the existence of several theories for social application that require commicating the diagnosis to identify them from the actors and their perceptions,
\end{abstract}

Rubén Téllez Peralta Licenciado en Ciencias de la Comunicación por la Universidad de Lima

Profesor de la Universidad Femenina del Sagrado Corazón. 
knowledge, level of information, attitudes, practices and components; also refers to the flexibility, an important characteristic during the planning of the diagnosis, so that it can conform to reality.

Keywords: social communication, diagnosis, culture, planning, implementation.

Hace más de 40 años, Abraham Moles (1970) identificó 250 definiciones de cultura, las más aceptadas de estas definiciones consideran a la cultura como la resultante histórica de los procesos de simbolización y representación de las relaciones e interacciones de un grupo humano con su medio físico y entorno social; y que afecta y caracteriza la cosmovisión, los comportamientos y las producciones de sus integrantes en sus diversas manifestaciones sociales: identidad, comunicación y lenguaje, valores y normas, hábitos y costumbres, política, administración del poder, estética y arte, tecnología y producción, intercambio de bienes... por ejemplo.

La cultura no es una práctica; ni es simplemente la suma descriptiva de los "hábitos y costumbres" de las sociedades, como tiende a volverse en ciertos tipos de Antropología. Está imbricada con todas las prácticas sociales, y es la suma de sus interrelaciones (Hall, Stuart; 1984).

Si definimos la comunicación como aquella dimensión cultural y social donde se construyen, comparten, negocian, apropian y atribuyen significados, se tiene que la relación entre cultura y comunicación es permanente, intensa y de coexistencia. Y es que, como bien dice Jesús Martín-Barbero (2008), la comunicación es "una dimensión constitutiva de la vida cultural y no solo su exhibición", es decir, que la comunicación no solo es la forma como se expresa la cultura sino además forma parte de ella.

Resulta imposible desde esta perspectiva separar acción-interacción-comunicación porque la comunicación se piensa como un proceso permanente y abierto de producción de sentido: La comunicación es el momento relacionante de la diversidad sociocultural simbólica y material. Una dimensión que atraviesa las otras dimensiones. (Massoni, Sandra; 2002). 
Este artículo trata sobre las relaciones que se dan entre la cultura y la comunicación en los procesos de diseño e implementación de los diagnósticos de comunicación.

Un diagnóstico tiene como finalidad producir conocimiento sobre una determinada realidad a fin de generar un cambio en ella; por lo tanto, en esencia, el diagnóstico es un instrumento aplicativo, no procura el conocimiento por el conocimiento, sino el conocimiento para la acción. En ese sentido, con el diagnóstico se busca comprender la realidad o situación de una organización, grupo social o colectividad, a fin de elaborar las propuestas, tomar las decisiones e implementar las alternativas más adecuadas para la modificación de dicha realidad.

El diagnóstico aplicado al campo de las comunicaciones recibe el nombre de Diagnóstico de Comunicación, y es el punto de partida del Planeamiento de la Comunicación y del diseño de Estrategias de Comunicación, ya que suministra la información y el análisis que ambos procesos requieren para poder desarrollarse y direccionar su actuación.

Pese a su importancia, el Diagnóstico de Comunicación no siempre es el punto de partida de las intervenciones sociales o de aquellas que tienen un componente comunicacional. Esto se explica -en unos casos- porque en algunas organizaciones la pertinencia comunicativa es considerada de forma instrumental, esto es, como la suma de actividades básicamente sencillas y estandarizadas orientadas al cumplimiento de protocolos y la producción de materiales de comunicación. Esta forma mecanizada y simplista de entender o desentender la comunicación, desactiva toda idea de estudiar o diagnosticar la situación de los procesos de comunicación y su influencia en las dinámicas institucionales, empresariales y sociales.

En otros casos, la comunicación no alcanza a ser percibida como una dimensión por sí misma relevante, y es incluida como un factor más dentro de diagnósticos organizacionales o comunitarios. En estos contextos, es usual que se refieran a los procesos de comunicación en términos de "canales de información, "flujos de información", "medios de comunicación"..., términos que tienen en común la ausencia de los sujetos y la sobrevaloración del medio y el mensaje.

Una tercera situación se da cuando durante la gestión, implementación o desarrollo de las actividades, quienes las conducen se percatan de la 
presencia de conductas disonantes, cuya explicación o solución son asociadas a la comunicación: indiferencia, apatía, tensión, rechazo, incumplimiento, insatisfacción, reclamo, confrontación, deserción... entre otras; y que generan situaciones o escenarios disfuncionales al logro de los objetivos y metas de la institución o empresa. Y es esta situación de crisis la que impulsa la necesidad de hacer un diagnóstico de comunicación.

\section{Rol de lo cultural en los diagnósticos de comunicación}

Siendo tan basto el campo de acción de "lo cultural" y siendo las comunicaciones una de las formas en que se expresa esa bastedad, lo que de alguna forma delimita la relación comunicación-cultura dentro de un Diagnóstico de Comunicación, son sus atributos de funcionalidad/ operatividad y enfoque.

Todo diagnóstico tiene un objetivo funcional: como se dijo antes, no se diagnostica para saber, sino para hacer; de manera que se van a privilegiar los aspectos culturales relacionados con la explicación de los procesos que se quieren afectar, es decir, la identificación de los aspectos críticos, sus causas, las barreras y los elementos facilitadores.

"El diagnóstico es el reconocimiento de un problema y sus posibles soluciones. En el ámbito de la comunicación implica detectar las necesidades sociales de comunicación presentes en determinadas condiciones, es decir, la diferencia entre la información necesaria y la disponible para la comprensión y orientación de la práctica social de individuos, grupos, estratos, clases, naciones" (Sandoval, Carlos; 1990).

De otro lado, el enfoque comunicacional de quiénes realizan o deciden sobre el diagnóstico, va a incidir en la mayor o menor presencia de los aspectos culturales en el diseño y aplicación del Diagnóstico de Comunicación. Por ejemplo, un diagnóstico de comunicación organizacional con enfoque administrativo, estará básicamente centrado en averiguar quiénes generan información, qué tipo de información, a través de qué canales, con qué frecuencia y propósito, quiénes reciben esa información y qué 
hacen con ella; desde este enfoque centrado en el flujo de información, el diagnóstico no incluye los tópicos relacionados con la satisfacción del trabajador, el clima laboral, el trabajo en equipo o la identificación con la institución, que sí los incluye -por ejemplo-, la perspectiva funcionalista contemporánea de la comunicación en las organizaciones.

En el campo empresarial, donde reina el marketing y su instrumento de diagnóstico: los estudios de mercado, el enfoque predominante es el del consumo; desde esta óptica los estudios básicamente indagan sobre el nivel de información, las actitudes y prácticas relacionadas con la recordación de marca, la intención de compra, la exposición a los medios y mensajes, las necesidades satisfechas y no satisfechas, las preferencias, las expectativas y las demandas de hombres y mujeres en tanto sujetos consumidores diferenciados demográfica (por nivel adquisitivo, sexo, edad, ocupación, lugar de residencia) y psicográficamente (actitudes hacia el consumo y estilo de vida). En este enfoque, la cultura es parte de lo que Loudon y Della Bitta (1995) llaman "factores ambientales que influyen en el comportamiento del consumidor" y que forman o dan forma a las percepciones y a los rituales y estilos de vida de los consumidores.

Desde una perspectiva cultural de la comunicación, se parte del supuesto de que la realidad es una construcción social que resulta de las relaciones intersubjetivas y procesos de simbolización que se dan en la vida cotidiana de los actores sociales, donde también existen matrices socioculturales y condicionamientos objetivos - provenientes de su modo de vida- que marcan sus cosmovisiones. A decir de Steigerwald (2008) "Esas matrices y condicionamientos objetivos se revelan en las representaciones sociales, las prácticas y las rutinas cotidianas de los actores.".

"(las representaciones) no están sólo en la cabeza: ellas organizan y regulan las prácticas sociales, influyen en nuestra conducta y consecuentemente tienen efectos prácticos, reales" (Hall, S., 1997 citado en Kenbel, Claudia, 2006).

Desde esta óptica, el Diagnóstico de Comunicación tiene como escenario de acción las mediaciones que se dan en la vida cotidiana de las personas, y centra su atención en identificar desde las rutinas, prácticas 
y representaciones sociales relacionadas con el objeto de estudio las matrices socioculturales que las generan.

El diagnóstico comunicacional parte de reconocer que la sociedad es un espacio de concertación entre diferentes grupos que perciben y actúan de maneras distintas porque funcionan desde matrices socioculturales diferentes. En este sentido, intenta, en vez de negar esta diversidad simbólica y material, recuperarla, porque es desde allí que se podrá trabajar en la articulación de intereses en función de un objetivo institucional u organizacional. (Massoni, Sandra; 2003).

En las últimas dos décadas, en el campo de la Comunicación para el Desarrollo, ha ido ganando mayor presencia el enfoque de la comunicación para el cambio de comportamiento (CCC), que a decir de Silvio Waisbord (2001) se introdujo con el Marketing Social para luego formar parte de otras propuestas y estrategias.

La comunicación para el cambio de comportamiento (CCC) es un proceso interactivo con las comunidades (integrado en un programa general) para desarrollar mensajes y enfoques adecuados usando una variedad de canales de comunicación para desarrollar comportamientos positivos; promover y sostener el cambio de comportamiento individual, de la comunidad y de la sociedad; y mantener comportamientos apropiados (FHI, 2003).

Este enfoque, a diferencia de las anteriores tendencias y modelos comunicacionales aplicados en nuestro país, centrados en la información/ difusión, en el mensaje/promoción y, en el desarrollo de conocimientos y competencias/educación-capacitación, sin negar ninguno de ellos, pone el énfasis en los comportamientos y el cambio, esto es, en la eficacia y resultados tangibles de los procesos de intervención comunicacional. Siendo varias las vertientes, las teorías sobre el comportamiento que más han influido en este enfoque son la Teoría de la Acción Razonada, la teoría del Aprendizaje Social y la teoría Transteórica (también conocida como la teoría del Cambio por Etapas). 
Estas teorías y los diversos modelos que se han generado para su aplicación social: modelo IEC (Información, Educación y Comunicación), modelo de Creencias en Salud, modelo Precede-Procede, modelo COMBI (Communication for Behavioural Impact), modelo ACMS (Abogacía, Comunicación y Movilización social), y muchos otros; de forma tácita o explícita, requieren que el Diagnóstico de Comunicación les permita identificar desde los actores y sus percepciones, el nivel de información, los conocimiento, las actitudes, las prácticas y los comportamientos que ellos/ellas tienen en relación al objeto de estudio.

Para ello, se necesita caracterizar al público objetivo/actor social, integrando a su perfil sociodemográfico (vivienda, ocupación, grado de instrucción...) un perfil sociocultural, que dé cuenta de los contextos, las prácticas y las percepciones que visibilizan a través de la palabra y otras expresiones culturales/comunicacionales sus representaciones sociales. En ese sentido, haciendo uso de técnicas cualitativas y cuantitativas, los diagnósticos de comunicación incluyen ejes temáticos como los que siguen:

- Formas de comunicación personal y comunal (recursos, capital comunicaional)

- Consumo de medios y preferencias

- Redes sociales o de socialización

- Rutina de vida (distribución del tiempo laboral, familiar, amical, de transporte...)

- Autopercepción y referentes de identidad

- Valoraciones (de prácticas, competencias, costumbres, relaciones, instituciones...)

- Creencias personales y normativas (norma subjetiva: la opinión de los otros)

- Experiencias previas

- Expectativas de vida

- Percepciones sobre las prácticas, contenidos, actores, situaciones y condiciones relacionadas con las conductas

Las propuestas de intervención en comunicación que apuestan por la participación y movilización social como impulsores del cambio comportamental, o que le dan a estos componentes un lugar privilegiado 
dentro de sus estrategias, necesitan que el Diagnóstico de Comunicación también ponga énfasis en identificar las redes sociales, los líderes, las instituciones, los medios de comunicación, las autoridades y los tomadores de decisión involucrados con el tema o situación a tratar. Y, además, que el diagnostico permita un mapeo de las actuaciones previas, el nivel de información y las actitudes de estos actores sociales hacia el tema, a efectos de definir adecuadamente sus públicos y aliados estratégicos.

\section{A modo de reflexión}

El Diagnóstico de Comunicación puede ser entendido como un instrumento, un método o una estrategia de conocimiento, esto es, como un recurso para obtener algo, como la forma de hacerlo o, como un escenario de búsqueda y reconocimiento de las lógicas de funcionamiento de las identidades, representaciones, tejidos y prácticas sociales.

Comprender la realidad desde los sujetos para poder aproximarse a los problemas, sus causas y posibles formas de darles solución no es una tarea fácil, se requiere de herramientas conceptuales y aplicativas que permitan aproximarse exitosamente a las simplicidades y complejidades del entramado social y de hombres y mujeres que no necesariamente quieren decir o saben cómo expresar aquello que viven. De allí que es acertado entender el Diagnóstico de Comunicación en su hacer como una estrategia: donde es pensada con minuciosidad y amplitud la claridad y viabilidad de los objetivos, la pertinencia del método, la funcionalidad de las técnicas, la adecuación de los contenidos e instrumentos a las características de los actores sociales y, la forma de acceder a ellos como personas y a sus mundos individuales y compartidos.

Y por más acuciosa que haya sido la planificación del diagnóstico, como toda estrategia debe ser flexible en su aplicación para ajustarse a la realidad y los nuevos escenarios que esta presenta.

La identificación de un problema, su formulación, la adecuada conceptualización, constituyen pasos necesarios para la realización de un diagnóstico de comunicación. Sin embargo, a medida que éste avanza, aquellos pueden ser ampliados, reformulados e incluso cuestionados 
totalmente. Sólo la dinámica de la investigación, realizada en el seno de la vida cotidiana de las comunidades, permite justificar la selección de un problema y el marco teórico aplicado. (Prieto, Daniel; 1985)

Está claro que los objetivos, métodos, técnicas y contenidos de los diagnósticos de comunicación van a variar según la mirada o enfoque que se tenga de la comunicación, las prácticas sociales, la cultura y las formas cómo los sujetos construyen/deconstruyen/reconstruyen sus representaciones sociales, y las ejercen en sus relaciones y actuaciones sociales día a día. Y debe estar igualmente claro que como soporte de toda acción comunicativa, donde incluimos el diagnóstico, hay principios que afirman su sentido social, como son: el respeto por las personas, sus identidades, sus ideas y saberes, su privacidad, su nombre, voz e imagen y los derechos que la constitución y los derechos humanos les reconocen.

Martín-Barbero afirma que no es posible entender la comunicación fuera de la cultura, y los diagnósticos de comunicación le dan la razón. Cada vez que se quiere profundizar sobre los procesos de comunicación, las líneas que le dan forma, los contextualizan y explican, provienen siempre, indefectiblemente, de la cultura. Con sus lógicas y matrices siempre inacabadas, donde coexisten lo individual, lo grupal, lo colectivo y lo masivo; lo tradicional y lo moderno; lo real y lo imaginado; lo masculino y lo femenino; lo propio y lo ajeno; lo que incluye y excluye; lo valorado y lo que no se tolera; lo aceptado y lo deseado; lo que provoca satisfacción, alegría, enojo, admiración, indignación, orgullo, adoración, esperanza... todo aquello que expresa la vida de las personas y que se construye socialmente pero se afirma culturalmente desde y con la comunicación.

\section{Referencias}

Family Health International. (2003) Comunicación para el cambio de comportamiento (CCC) para el VIH/SIDA. Un marco de trabajo estratégico.

Hall, Stuart. (1984) Cultural Studies: two paradigms. Media, Culture and Society, 2, Londres, 1980. Traducción al castellano en: Hueso húmero, no 19. Lima. 
Martín- Barbero, Jesús. (1987) De los medios a las mediaciones. Comunicación, cultura y hegemonía. Barcelona.

Martín- Barbero, Jesús. (2002) Oficio de cartógrafo, travesias latinoamericanas de la comunicación en la cultura. Santiago de Chile.

Massoni, Sandra. (2002) Diagnóstico comunicacional sobre las percepciones diferenciales del problema de la sostenibilidad del sistema agrícola en Casilda. Revista de investigaciones de la Facultad de Ciencias Agrarias. Univ. Nacional de Rosario.

Massoni, Sandra. (2003) Estrategias de comunicación rural: un modelo de abordaje de la dimensión comunicacional para el desarrollo sostenible entendido como cambio social. Tesis doctoral Univ. de Buenos Aires.

Prieto, Daniel. (1985)Diagnóstico de Comunicación. Manuales de CIESPAL, Editorial Belén, Quito, Ecuador.

Sandoval, Carlos. (1990) "Planificar la Comunicación: Conceptos, Herramientas, Desafios". En Contribuciones N.6, Instituto de Investigaciones Sociales de la Universidad de Costa Rica, San José, Costa Rica.

Steigerwald, María Alejandra. (2008) Comunicación y cultura un abordaje desde las mediaciones y representaciones sociales. Ponencia. Salta, Argentina.

Uranga, Washington. (2001) Mirar desde la comunicación. Una forma de analizar las prácticas sociales. Buenos Aires.

Waisbord, Silvio. (2001) Árbol genealógico de teorías, metodologías y estrategias en la comunicación para el desarrollo. Fundación Rockefeller.

Correo electrónico: ruben_tellez@yahoo.es 